\title{
Development of a Spectral Model Based on Charge Transport for the Swift/BAT 32K CdZnTe Detector Array
}

\author{
Goro Sato ${ }^{a, b, *}$ Ann Parsons ${ }^{c}$ Derek Hullinger ${ }^{c, d}$ Masaya Suzuki ${ }^{e}$ \\ Tadayuki Takahashi ${ }^{\text {a,b }}$ Makoto Tashiro ${ }^{\mathrm{e}}$ Kazuhiro Nakazawa ${ }^{\mathrm{a}}$ \\ Yuu Okada ${ }^{b}$ Hiromitsu Takahashi ${ }^{b}$ Shin Watanabe ${ }^{a}$ \\ Scott Barthelmy ${ }^{c}$ Jay Cummings ${ }^{\text {c,f }}$ Neil Gehrels ${ }^{c}$ Hans Krimm ${ }^{c, f}$ \\ Craig Markwardt ${ }^{\mathrm{c}, \mathrm{d}}$ Jack Tueller ${ }^{\mathrm{c}}$ Ed Fenimore ${ }^{\mathrm{g}}$ David Palmer ${ }^{\mathrm{g}}$ \\ ${ }^{2}$ Institute of Space and Astronautical Science (ISAS) / JAXA, 3-1-1 Yoshinodai, \\ Sagamihara, Kanagawa 229-8510, Japan \\ ${ }^{\mathrm{b}}$ University of Tokyo, 7-3-1, Hongo, Bunkyo, Tokyo 113-0033, Japan \\ ${ }^{\mathrm{c}}$ NASA / Goddard Space Flight Center, Code 661, Greenbelt, MD 20771, USA \\ ¿University of Maryland, College Park, MD 20742, USA \\ 'Saitama University, 255 Shimo-Okubo, Sakura, Saitama 338-8570, Japan \\ f Universities Space Research Assosiation (USRA), Seabrook, MD, USA \\ g Los Alamos National Laboratory, Los Alamos, NM, USA
}

\begin{abstract}
The properties of $32 \mathrm{~K} \mathrm{CdZnTe}\left(4 \times 4 \mathrm{~mm}^{2}\right.$ large, $2 \mathrm{~mm}$ thick) detectors have been studied in the pre-flight calibration of the Burst Alert Telescope (BAT) on-board the Swift Gammaray Burst Explores (scheduled for launch in November 2004). In order to understand the energy response of the BAT CdZnTe array, we first quantify the mobility-lifetime $(\mu \tau)$ products of carriers in individual $\mathrm{CdZnTe}$ detectors, which produce a position dependency in the charge induction efficiency and results in a low energy tail in the energy spectrum. Based on a new method utilizing ${ }^{57} \mathrm{Co}$ spectra obtained at different bias voltages, the $\mu \tau$ for electrons ranges from $5.0 \times 10^{-4}$ to $1.0 \times 10^{-2} \mathrm{~cm}^{2} V^{-1}$ while the $\mu \tau$ for holes ranges from $1.3 \times 10^{-5}$ to $1.8 \times 10^{-4} \mathrm{~cm}^{2} V^{-1}$. We find that this wide distribution of $\mu \tau$ products explains the large diversity in spectral shapes between $\mathrm{CdZnTe}$ detectors well. We also find that the variation of $\mu \tau$ products can be attributed to the difference of crystal ingots or manufacturing harness. We utilize the $32 \mathrm{~K}$ sets of extracted $\mu \tau$ products to develop a spectral model of the detector. In combination with Monte Carlo simulations, we can construct a spectral model for any photon energy or any incident angle.
\end{abstract}

Key words: Gamma-ray astronomy, Swift, BAT, CdZnTe, mobility-lifetime products, spectral model 


\section{Introduction}

Swift, scheduled for launch in November 2004, is a NASA MIDEX mission dedicated to the study of astronomical gamma-ray bursts (GRBs) [1]. It carries the Burst Alert Telescope (BAT) which will detect and localize the GRBs. The BAT is a coded aperture instrument with a 1.4 steradian field-of-view (half coded) (shown in Fig. 1). The detector plane is an assembly of 32,768 planar Cadmium Zinc Telluride (CdZnTe, CZT) detectors each $4 \times 4 \mathrm{~mm}^{2}$ and $2 \mathrm{~mm}$ thick. They form a $1.2 \mathrm{~m} \times 0.6 \mathrm{~m}$ sensitive area, and provide imaging capability and spectroscopy [2]. In addition to detecting GRBs, BAT will also conduct a sensitive hard X-ray all-sky survey over the course of the mission.

Recently, Cadmium Telluride ( $\mathrm{CdTe}$ ) and CdZnTe semiconductor materials have attracted much attention as hard X-ray and gamma-ray detectors among the semiconductor materials with a wide band gap. Their good energy resolution and the fact that these can be fabricated into compact arrays make them very attractive in comparison with inorganic scintillation detectors coupled to either photodiodes or photo-multiplier tubes (e.g. [3]). Because of these advantages, a large CdTe gamma-ray camera is already operating in space for the International Gamma Ray Astrophysics Laboratory (INTEGRAL) mission. It is also a coded aperture instrument which utilizes 16,384 planar CdTe $\left(4 \times 4 \mathrm{~mm}^{2}, 2 \mathrm{~mm}\right.$ thick) detectors to form a sensitive area of $2,621 \mathrm{~cm}^{2}$. This provides high resolution spectroscopy with an energy resolution of $9 \%$ (FWHM) at $100 \mathrm{keV}$ and fine imaging with an angular resolution of $12 \operatorname{arcmin~[4]~[5].~}$

However, despite recent advances, the considerable charge loss in CdTe and CdZnTe limits their capability as high resolution spectrometers. Due to the low mobility and the short lifetime of the carriers, the electron and hole pairs generated by gamma-ray irradiation cannot be fully collected. As a result, a broad low energy tail is seen in the detector's energy spectrum. In the case of CdZnTe material, it is also known that the current High Pressure Bridgman (HPB) technique produces only polycrystals with a non-uniform distribution of charge transport properties. This can result in some degree of variation in charge transport properties between CdZnTe detectors. Therefore, it is necessary to measure these properties for each $\mathrm{CdZnTe}$ detector in order to understand the BAT energy response.

In this paper, we report the features of individual CdZnTe spectra. Then we evaluate the key mobility and lifetime parameters both for electrons and holes. Finally, we describe how these are used to develop the spectral model for the individual detectors and for the entire CdZnTe array.

\footnotetext{
* Corresponding author. Tel.: +81-42-759-8545; fax: +81-42-759-8546

Email address: gsato@astro. isas.jaxa.jp (Goro Sato).
} 


\section{CdZnTe array in the Burst Alert Telescope}

The BAT detector plane $\left(5,243 \mathrm{~cm}^{2}\right)$ is composed of 32,768 pieces of $4 \mathrm{~mm} \times 4 \mathrm{~mm} \times 2 \mathrm{~mm}$ $\mathrm{CdZnTe}$ detector elements. Groups of 128 detector elements are assembled into $8 \times 16$ subarrays. The signals from each of the 128 detectors are read out by a single analog signal processing ASIC (XA1). This 128-channel XA1 readout chip was designed and produced by IDEAS (Hovik, Norway). A Detector Module (DM) contains two such sub-arrays, and a block is made of eight DMs. There are in total 16 blocks mounted in the Detector Array Plane.

The pulse heights are digitized by an ADC unit on the XA1 and transmitted to a data handling system along with the channel ID for the event. Thus, the events are accumulated into individual spectra for each CdZnTe pixel. There is an electronic calibration pulser circuit within each DM which can sequentially inject a specified number of charge pulses of a specified level into each of the 128 channels. The XA1 has a dynamic range up to $\sim 500 \mathrm{keV}$ which is not perfectly linear, and we can correct the offset, gain, and nonlinearity of each of the $32 \mathrm{~K}$ detectors with the use of this calibration pulser.

The $\mathrm{CdZnTe}$ array will have a nominal operating temperature of $20^{\circ} \mathrm{C}$ and its thermal gradients (temporal and spatial) will be kept to within $\pm 1{ }^{\circ} \mathrm{C}$. The typical bias voltage is $200 \mathrm{~V}$, with a maximum of $300 \mathrm{~V}$. The $\mathrm{CdZnTe}$ material used in the BAT is manufactured by eV Products Inc. (Saxonburg, PA) using the HPB technique. All detectors were tested by the manufacturer to meet minimum performance specifications. In addition to mechanical and visual inspections, the detector performance specifications listed in Table 1 were required for the energy spectrum from a ${ }^{57} \mathrm{Co}$ radioactive source. A typical ${ }^{57} \mathrm{Co}$ spectrum obtained with a single CdZnTe detector is shown in Fig. 2. We can see 14.4, 122 and $136 \mathrm{keV}$ lines and also a prominent low energy tail which accompanies the $122 \mathrm{keV}$ line. Small humps around $100 \mathrm{keV}$ are escape peaks associated with the $122 \mathrm{keV}$ main peak, which are due to the escape of photo-fluorescence photons (23 keV for $\mathrm{Cd}$ and $27 \mathrm{keV}$ for $\mathrm{Te}$ ) from the detector. As described in the Introduction, the charge transport properties of the CdZnTe material were the major issue for the selection of $\mathrm{CdZnTe}$ detectors. Therefore, peak-to-valley ratios calculated at 122 and $14.4 \mathrm{keV}$ lines were used in the screening.

\section{Spectral Properties}

The intensive pre-flight calibration has been performed at the NASA/Goddard Space Flight Center [6]. The calibration is divided into two phases. In the first phase, measurements were made with each block individually. This was carried out right after the blocks were constructed. In the second phase, measurements were made with the entire array inside a large clean tent, after all sixteen blocks were installed on the detector array plate. In order to understand the BAT instrument's response to incident $15-150 \mathrm{keV}$ gamma-rays, we acquired spectra using various radioactive sources (namely, ${ }^{241} \mathrm{Am},{ }^{57} \mathrm{Co},{ }^{109} \mathrm{Cd},{ }^{133} \mathrm{Ba}$ and ${ }^{137} \mathrm{Cs}$ ) at different bias voltages, temperatures and incident angles. 


\subsection{Properties of Individual CdZnTe Spectra}

Even after correction for electronics gain, offset and nonlinearity, we still observe a significant variation in peak channels as can be seen in Fig. 3 (a), which shows a distribution of the peak channels of the $122 \mathrm{keV}$ line extracted from $32 \mathrm{~K}$ spectra. The data were obtained during the block calibration with a ${ }^{57} \mathrm{Co}$ source placed $1 \mathrm{~m}$ above the center of the block detector plane. The detectors were operated with a bias voltage of $200 \mathrm{~V}$ at a temperature of $20^{\circ} \mathrm{C}$.

The peak channel varies more than $10 \%$ from detector to detector. We consider this to be a result of differences in charge induction efficiency between detectors. The peak-to-valley ratio is another parameter which characterizes the charge transport properties. We define it as the ratio of counts at $122 \mathrm{keV}$ to counts at $100 \mathrm{keV}$ where the counts at these energies are averages over 5 neighboring channels. As shown in Fig. 3 (b), the peak-to-valley ratio ranges from 2 to 4.

The energy resolutions measured at $122 \mathrm{keV}$ are shown in Fig. 3 (c) along with those at $22 \mathrm{keV}$ derived from ${ }^{109} \mathrm{Cd}$ spectra. The average value of FWHM at $122 \mathrm{keV}$ is much greater than at $22 \mathrm{keV}$, even after taking into account the expected increase due to Fano noise. This is because the $122 \mathrm{keV}$ peak has an asymmetric shape due to the low energy tail, which is prominent only for higher energy lines. The wider distribution of FWHM at $122 \mathrm{keV}$ is due to the variation in the shape of low energy tail.

\subsection{Summed Spectrum for the Entire CdZnTe Array}

The variation of peak channel shown in $\S 3.1$ indicates that it is not sufficient to correct only for the readout electronics when we construct a summed spectrum for the entire CdZnTe array. The shift of photo-peak due to the charge transport properties in CdZnTe materials should also be corrected. For this, we simply use the actual peak channels of the 22.1, 59.5, 88.0 and $122.1 \mathrm{keV}$ lines. The peak channels are fitted well with a quadratic function of energy, which is used to convert the ADC channel to energy for each $32 \mathrm{~K} \mathrm{CdZnTe} \mathrm{pixel.}$

To obtain the ${ }^{57} \mathrm{Co}$ summed spectrum, we acquire data at the level of the array calibration, in which the detector array is located $1 \mathrm{~m}$ below the coded aperture mask. The ${ }^{57}$ Co source is placed at $3 \mathrm{~m}$ above the center of the detector plane, or $2 \mathrm{~m}$ above the center of the coded aperture mask. Therefore, the detector signals are modulated by the mask pattern. To obtain the summed spectrum, we also applied the "mask weighting" method, which utilizes the mask modulation (i.e., how much the detectors are shadowed) as summation weights [7]. This enables us to eliminate flat non-modulated components such as scattering. The summed ${ }^{57} \mathrm{Co}$ spectrum obtained with this method is shown in Fig. 4. The 122 and $136 \mathrm{keV}$ lines are clearly seen. The energy resolution is $6.7 \mathrm{keV}$ (FWHM) at $122 \mathrm{keV}$. 


\section{Charge Transport Properties in Individual CdZnTe Detectors}

\subsection{Fitting Procedure to Extract $\mu \tau$ products}

The mobility and lifetime for electrons and for holes are key parameters when we model the spectral response of a $\mathrm{CdZnTe}$ detector, because once we know these values, the charge induction efficiency can be calculated at each depth in the detector and applied to any incident photon energy. Here we deduce $(\mu \tau)_{\mathrm{e}}$ and $(\mu \tau)_{\mathrm{h}}$ for each detector by following the concept described in Sato et al.[8]. We first derive a spectral fitting function to be applied to the $122 \mathrm{keV}$ peak in the ${ }^{57} \mathrm{Co}$ spectrum. We assume the ideal situation in which the electric field $(E)$ throughout the detector volume is constant. Then the charge induction efficiency is given by the Hecht equation [9]

$$
\begin{aligned}
& \eta(x)=\frac{(\mu \tau)_{\mathrm{e}} E}{D} {\left[1-\exp \left(-\frac{D-x}{(\mu \tau)_{\mathrm{e}} E}\right)\right] } \\
&+\quad \frac{(\mu \tau)_{\mathrm{h}} E}{D}\left[1-\exp \left(-\frac{x}{(\mu \tau)_{\mathrm{h}} E}\right)\right]
\end{aligned}
$$

where $D$ is the detector thickness and $(\mu \tau)_{\mathrm{e}}$ and $(\mu \tau)_{\mathrm{h}}$ are mobility-lifetime products for electrons and holes, respectively. It is described as a function of the interaction depth $(x)$ from the cathode surface for a specific set of $\mu \tau$ products.

The pulse height from $\mathrm{CdZnTe}$ depends on the depth where the interaction takes place. Therefore, when we construct a spectral model, we need to know the "depth distribution", which indicates how many photons deposit energy at different depths in a detector. For this, we perform a Monte Carlo simulation, which can also incorporate the photo-fluorescence ( $23 \mathrm{keV}$ for $\mathrm{Cd}$ and $27 \mathrm{keV}$ for Te) properly. The obtained distribution is shown in Fig. 5 as a function of the depth from the cathode surface, $\frac{\mathrm{d} P(x)}{\mathrm{d} x}$. After multiplying it by a certain small thickness $\mathrm{d} x$, it gives the probability that a photon will deposit all of its energy (producing the main photo-peak) within $\mathrm{d} x$ at each depth $x$ for an unit photon flux (photons $/ \mathrm{cm}^{2}$ ) incident normal to the top of the detector. In addition to the $122 \mathrm{keV}$ line, we obtain the distribution for the $136 \mathrm{keV}$ line because a considerable amount of its low energy tail impinges on the energy region of $122 \mathrm{keV}$ line. We can see the decrease of full energy deposits at both ends of a detector, which is due to the escape of photo-fluorescence photons from the detector surface.

The detector response for monochromatic gamma-rays is generated in the following way. We first divide a detector into a number of slices. The relative pulse height is calculated from the Hecht equation at each slice with respect to a certain channel, called $C H_{\max }$. We fill the model histogram at the calculated pulse height channel with the interaction probability given by the "depth distribution". This operation is iterated for all the slices from the cathode side to the anode side, which is schematically shown as follows: 


$$
\begin{aligned}
& \sum_{x=\text { cathode }}^{\text {anode }} \text { Fill }\left(C H_{\max } \times \eta(x), \frac{\mathrm{d} P(x)}{\mathrm{d} x} \times \mathrm{d} x\right) \\
& \Longrightarrow f\left(E_{\gamma},(\mu \tau)_{\mathrm{e}} ;(\mu \tau)_{\mathrm{h}}, E, \sigma, C H_{\max }\right)
\end{aligned}
$$

where $E_{\gamma}$ is the energy of incident gamma-rays and $\sigma$ is the resolution excluding the tail structure. The resultant spectrum gives the peak shape and is determined by the 6 parameters listed in the above equation. While the product of $(\mu \tau)_{\mathrm{e}} \times E$ mainly determines the peak channel, the product of $(\mu \tau)_{\mathrm{h}} \times E$ determines the amount of the low energy tail. Since the parameter $\mathrm{CH}_{\max }$, the channel where Hecht equation becomes unity, is unknown beforehand and cannot be determined from a single spectrum, we acquire ${ }^{57} \mathrm{Co}$ spectra at three different bias voltages $(100,150,200 \mathrm{~V})$ to utilize the peak shifts due to the changes in the strength of electric field $(E)$. Then, we simultaneously fit the three spectra around the $122 \mathrm{keV}$ main peaks with free parameters of a single set of $\mu \tau$ for electrons and $\mu \tau$ for holes, $C H_{\max }$, and also 3 sets of $\sigma$ and normalization (i.e. 9 free parameters in total). This therefore allows us to extract the $\mu \tau$ products from the individual detectors. It is necessary to use the data corrected for electronics gain, offset and nonlinearity in the way described in $\S 3.1$. To take into account the $136 \mathrm{keV}$ line component with its low energy tail extending to the energy region of the $122 \mathrm{keV}$ line, the response for the $136 \mathrm{keV}$ line is also calculated using the same parameters for $122 \mathrm{keV}$ except for $E_{\gamma}$, and then added to the models.

We apply the model fitting to a single CdZnTe detector. In the model calculation, the number of slices is chosen to be 1000 to calculate the "depth distribution" precisely to the thickness of $2 \mu \mathrm{m}$. The region used in the fit is selected above $107 \mathrm{keV}$ to eliminate the escape peaks and to minimize the effect of the scattered component. The region is also restricted to the bins which contain over 10 counts for statistics. This usually excludes the $136 \mathrm{keV}$ peak (but includes its tail component lying under the $122 \mathrm{keV}$ component). For the particular detector shown in Fig. 6, $\mu \tau$ for electrons and $\mu \tau$ for holes are determined as $2.57 \pm 0.03 \times 10^{-3}$ and $5.80 \pm 0.33 \times$ $10^{-5} \mathrm{~cm}^{2} \mathrm{~V}^{-1}$, respectively, with $\chi^{2} / \nu=241 / 244$. As shown in Fig. 6 , it is clear that the shift of the peak channel and the change of the low energy tail with respect to the change of bias voltage can be reproduced by our model.

We implemented our fitting procedure using the ROOT analysis tool kit[10]. The $\chi^{2}$ fitting is performed by the MINUIT package in ROOT. It takes $\sim 2$ minutes to fit a set of spectra for each detector with an AMD Athlon(TM) XP 3000+ machine.

\subsection{Finding $\mu \tau$ products for the $32 \mathrm{~K}$ CdZnTe detectors}

As indicated in $\S 3.1$, the diversity in spectral shape is produced by the variation in the charge transport properties between $\mathrm{CdZnTe}$ detectors. Here, we evaluate such variations in terms of $\mu \tau$ products by applying the spectral fitting method to all the $32 \mathrm{~K} \mathrm{CdZnTe}$ detectors.

In the individual block calibration, we acquired ${ }^{57} \mathrm{Co}$ spectra at the three different bias voltages $(100,150,200 \mathrm{~V})$. The temperatures were maintained at $20^{\circ} \mathrm{C}$, though, because of the limited calibration time, two of the blocks had to be operated at $10^{\circ} \mathrm{C}$. In order to minimize the scattered 
component in the spectra, the measurements were performed in such a way that the radioactive source was mounted inside a box made of lead with a hole cut in it large enough to irradiate all $2048 \mathrm{CdZnTe}$ detectors in the block. A large shielding plate was attached to the box to prevent photons scattering off the room's ceiling. Then, we applied the fitting method to $122 \mathrm{keV}$ peaks for the 32K CdZnTe detectors. We successfully extracted 29,347 ( 90\%) sets of $\mu \tau$ products as shown in Fig. 7. The two parameters exhibit remarkably wide distributions, ranging over more than 1 order of magnitude, showing a slight correlation. The $\mu \tau$ for electrons ranges from $5.0 \times 10^{-4}$ to $1.0 \times 10^{-2} \mathrm{~cm}^{2} \mathrm{~V}^{-1}$, and $\mu \tau$ for holes ranges from $1.3 \times 10^{-5}$ to $1.8 \times$ $10^{-4} \mathrm{~cm}^{2} \mathrm{~V}^{-1}$. Typical errors are $3.5 \%(1 \sigma)$ and $8.7 \%(1 \sigma)$ for the $\mu \tau$ for electrons and $\mu \tau$ for holes, respectively.

Examples of the extreme differences in the spectra due to the different $\mu \tau$ products are shown in Fig. 8, where the symbols "a" to "d" correspond to the location in Fig. 7. Though the selected detectors are exposed to the ${ }^{57}$ Co source for the same duration, there are large differences in spectral shapes. Detectors (a) and (b) have larger values of the $\mu \tau$ for holes than detectors (c) and (d), and their spectra show higher $122 \mathrm{keV}$ peaks than those of detectors (c) and (d). In contrast, there are more counts in the lower energy tail in the spectra (c) and (d). The more interesting feature is that spectrum (a) with a smaller value of $\mu \tau$ for electrons exhibits a higher $122 \mathrm{keV}$ peak than spectrum (b). Likewise, spectrum (c) has a higher peak than spectrum (d). To investigate this relation between the $\mu \tau$ products and the spectral shapes, we use the measured peak-to-valley ratios at $122 \mathrm{keV}$, which are calculated in section 3.1. We first subdivide the $\mu \tau$ products cross-plot into grids with equal log-scale intervals. Then, the peak-to-valley ratios are averaged over the detectors in each grid and shown in grey-scale in Fig. 9. First, it is evident that the peak-to-valley ratio increases as the $\mu \tau$ for holes becomes higher. Second, the detectors with largest values of peak-to-valley ratio are found in top and left edge of the $\mu \tau$ distributions. This trend can be recognized as a result of the closer values between $\mu \tau$ for electrons and $\mu \tau$ for holes. In such a detector, there will be a smaller difference in charge induction efficiency throughout the detector, and as a result, a higher $122 \mathrm{keV}$ photo-peak can be formed in the spectrum.

Furthermore, we find that the extracted $\mu \tau$ products are somewhat correlated to the DM or block location [11], which turns out to be strongly correlated to the detector boule number. In Fig. 10, the $\mu \tau$ products for $\sim 8,000$ detectors are shown against detector number sorted by ingot ID. It is realized that the diversities of $\mu \tau$ products can be attributed to the difference of crystal ingots or manufacturing harness, though there remains a large variation in the $\mu \tau$ for holes even in the same ingots.

\subsection{Temperature Dependence of $\mu \tau$ products}

The carrier mobilities are intrinsically dependent on temperature, limited by phonon scattering at higher temperatures and by impurity scattering at lower temperatures. This dependence in HPB CdZnTe has been reported in several papers [12]. However, since it is highly dependent on material qualities, it is not clear how the carriers in BAT CdZnTe detectors behave with temperature. Thus, for one specific block $(2,048 \mathrm{CdZnTe}$ detectors), we take the same fitting 
procedure to extract $\mu \tau$ products from ${ }^{57} \mathrm{Co}$ spectra acquired at lower temperatures of 0 and $10^{\circ} \mathrm{C}$ in addition to the data at $20^{\circ} \mathrm{C}$.

The distributions of extracted $\mu \tau$ products are shown in Fig. 11. There are trends in both distributions with the $\mu \tau$ product shifting slightly downward with a decrease in temperature, but the overall shape is not affected very much. For individual detectors, the relative values of $\mu \tau$ products are calculated with respect to the values at $20^{\circ} \mathrm{C}$. Since we observe a large variation from detector to detector in the way that $\mu \tau$ products change from 20 to 10 , and $0^{\circ} \mathrm{C}$, we show the averaged values and the RMS variations between detectors in Fig. 12 after subtraction of the deviation due to $\mu \tau$ extraction errors. We find that the $\mu \tau$ for electrons decreases by $\sim 9 \%$ while the $\mu \tau$ for holes decrease by $\sim 29 \%$ from 20 to $0{ }^{\circ} \mathrm{C}$ on average.

\section{Development of the Spectral Model}

Our final goal is to develop an energy and angular response for the entire CdZnTe array from 15 to $150 \mathrm{keV}$ and from 0 to $60^{\circ}$ for a large FOV of the BAT instrument. Using the model calculations described in the previous section, it is possible to calculate the pulse heights for any incident photon energy and to obtain the "depth distribution" for any incident angle with the use of Monte Carlo simulations.

\subsection{Energy and Angular Response for a Single CdZnTe Spectrum}

\subsubsection{Response for the $59.5 \mathrm{keV}$ Line}

We first calculate a model function for $59.5 \mathrm{keV}$ to be applied to an ${ }^{241} \mathrm{Am}$ spectrum obtained with a specific CdZnTe detector as shown in Fig. 13. In the 6 parameters of the model function (eq. 2), the $\mu \tau$ products are set to the values determined through the spectral fitting of the $122 \mathrm{keV}$ line while the $\sigma$ and $C H_{\max }$ are allowed to vary to adjust the resolution and the gain. We can see the model reproduces the asymmetric shape of photo-peak very well from $\sim 55$ to $\sim 64 \mathrm{keV}$. However, there remains an excess over the tail structure below $55 \mathrm{keV}$, which is observed in most of the detectors. After a more detailed study, we determined that this is most likely due to the combination of Compton scattering from a plastic package surrounding the radioactive source and an illumination onto the side surface of a detector, which impinges on deep regions below the cathode surface where the charge induction efficiency is relatively low. Another possibility is a non-uniformity in the $\mu \tau$ products within a detector. Suzuki et al. [13] have reported that there exist variations in $\mu \tau$ products on scales less than $1 \mathrm{~mm}$. Here it should also be noted that the lower part of the tail component is somewhat uncertain because the $\mu \tau$ products are extracted with the fitting of the restricted energy region around the main peaks (see Fig. 6). 


\subsubsection{Response for Low Energy Lines}

The response for lower energy lines are investigated with a ${ }^{109} \mathrm{Cd}$ spectrum. We calculate model functions for 22 and $25 \mathrm{keV}$ lines to be almost Gaussians because most of the interactions occur in shallow regions below the cathode surface. However, we observe a low energy tail component below $20 \mathrm{keV}$ as shown in Fig. 14. To evaluate the low energy tail, we introduce an empirical formula:

$$
f(x) \propto \exp \left(\frac{x-x_{\text {peak }}}{\lambda}\right) \text { if } x<x_{\text {peak }}
$$

where $x$ and $x_{\text {peak }}$ are the energy and peak channel, and $\lambda$ is a parameter to determine how the tail decreases with separation from the main peak. The component is added to the model after smearing with the energy resolution, which is fitted well with the actual data (Fig. 14). It is partly explained with side illumination onto the side surface of the detector and with Compton scattering from the plastic tablet of the radioactive source. However, if the incident photon energy is higher than the K-edges of $\mathrm{Cd}$ and Te $(26.7$ and $31.8 \mathrm{keV})$, such as the 31 and $35 \mathrm{keV}$ lines from a ${ }^{133} \mathrm{Ba}$ source, the situation becomes more complicated because escape photons from adjacent detectors contaminate the energy region below the main peaks.

\subsubsection{Angular Response}

The "depth distribution" depends on the incident angle. Fig. 15 (left) demonstrates how the distribution changes from an incident angle of 0 to 30 and $60^{\circ}$. The incident angle affects the energy response in two main ways: one is a decrease of the effective area, and the other is a decrease of the interaction depth. This changes the shape and amount of the low energy tail as presented in Fig. 15 (right).

\subsubsection{Modeling the Escapes}

Another important feature in CdZnTe spectra is "escape peaks", which occur as a result of photo-fluorescence. The prominent peaks are of $\mathrm{Cd}-\mathrm{K}$ and Te-K escapes, which appear at 23 and $27 \mathrm{keV}$ below main photo-peak, respectively. To model the escape peaks, we count the events where the energy depositions are smaller by 23 or $27 \mathrm{keV}$ than the full incident photon energy and derive the "depth distributions" for escape peaks, which are shown in Fig. 16 (left). Then, by applying the charge induction efficiency such as with the main photo-peak, we can obtain the model of escape peaks as shown in Fig. 16 (right).

\subsection{Composite Model for the Entire CdZnTe Array}

To develop the spectral model for the entire CdZnTe array, it is necessary to take into account the large range of $\mu \tau$ products. For this, we first divide the $32 \mathrm{~K}$ detectors into 9 -by- 7 groups 
with equal log-scale intervals. The percentage of detectors included in each grid is shown in Fig. 17. Then, we construct spectral models with $\mu \tau$ products at the center of each grid. The models are calculated for the most populated groups with the criteria of $>0.1 \%$. Thus, there are 35 spectral models based on the model functions developed for single CdZnTe detectors. Finally, we sum them, using the fraction of detectors as a weight. This gives us a composite spectral model which represents the energy response for the entire $\mathrm{CdZnTe}$ array.

The resultant spectral model is shown in Fig. 18 with an actual calibration spectrum resulting from a ${ }^{57}$ Co source positioned slightly off-axis. Although there still remains the discrepancy in the lower part of the tail, the model represents the overall spectral shape very well. To demonstrate how the model can reproduce the summed spectrum over a wide energy range, we construct a spectral model for ${ }^{133} \mathrm{Ba}$ spectrum, which includes $31,35,53$ and $81 \mathrm{keV}$ lines. The result is shown in Fig. 19, which looks almost perfect. The model reproduces the tail component given by the $\mu \tau$ products, the tail component for low energy lines, and also escape peaks. Note that the values of the $\mu \tau$ products of electrons and holes are fixed at those values obtained in $\S 4$.

\section{Conclusion}

Based on the fitting method applied to ${ }^{57} \mathrm{Co}$ spectra, we have succeeded to extract $\mu \tau$ products both for electrons and holes from $32 \mathrm{~K}$ individual $\mathrm{CdZnTe}$ detectors. The reason for this procedure is to calculate a spectral model following the actual physical processes from the photon-detector interaction to the charge transport. Once the $\mu \tau$ products are obtained, it allows us to predict the energy response for any photon energy or any incident angle. The actual calibration data is reproduced well by the model calculated with the Hecht equation and the interaction depth distributions for each energy. However, since various structures can be produced by scattered components and other geometrical effects, it is also necessary to estimate carefully such contributions with the use of Monte Carlo simulations in each case. Another important point is to deal with the large distributions of $32 \mathrm{~K}$ sets of $\mu \tau$ products. By grouping the detectors, we have successfully developed the spectral model for the entire CdZnTe array, which is to be included in the software of the Detector Response Matrix (DRM) generator for the BAT instrument.

\section{Acknowledgments}

We are grateful to P. G. Edwards for critical reading of this manuscript. G. S. is supported by the Research Fellowships of the Japan Society for the Promotion of Science for Young Scientists (14-08554). This research was supported by the NASA Swift mission and in part by the Ministry of Education, Science, Sports and Culture, Grant-in-Aid in Japan (13304014, 14079207). 


\section{References}

[1] Gehrels, N. et al., 2004, ApJ, 611, 1005

[2] Barthelmy, S., 2003, proc. SPIE, 5165, 175

[3] Takahashi, T. \& Watanabe, S., 2001, IEEE Trans. Nucl. Sci., 48, 950

[4] Ubertini, P. et al., 2003, A\&A, 411, L131

[5] Lebrun, F. et al., 2003, A\&A, 411, L141

[6] Parsons, A. et al., 2003, proc. SPIE, 5165, 190

[7] Hullinger, D. et al., 2003, proc. SPIE, 5198, 225

[8] Sato, G. et al., 2002, IEEE Trans. Nucl. Sci., 49, 1258

[9] Hecht, K., 1932, Zeits. Phys., 77, 235

[10] http://root.cern.ch/

[11] Sato, G. et al., 2003, proc. SPIE, 5198, 209

[12] Suzuki, K. et al., 2002, IEEE Trans. Nucl. Sci., 49, 1287

[13] Suzuki, M. et al., 2004, IEEE Trans. Nucl. Sci., in press 
Table 1

Required detector performance specifications. Peak-to-valley ratio at $14.4 \mathrm{keV}$ is defined as $\mathrm{Peak}_{14} / \mathrm{L}_{\text {Valley }}$ where $\mathrm{Peak}_{14}=$ average counts from 3 channels around $14.4 \mathrm{keV}$ and $\mathrm{L}_{\text {Valley }}=$ average counts from 3 channels in the valley on the left of the $14.4 \mathrm{keV}$ peak. Peak-to-valley ratio at $122 \mathrm{keV}$ is defined as Peak ${ }_{122} /$ Peak $_{100}$ where Peak ${ }_{122}$ and Peak 100 are averages counts from 3 channels around 122 and $100 \mathrm{keV}$, respectively.

\begin{tabular}{ll}
\hline Detector performance & Requirement \\
\hline Peak-to-valley ratio at $14.4 \mathrm{keV}$ & $>4.5$ \\
Peak-to-valley ratio at $122 \mathrm{keV}$ & $>2.5$ \\
FWHM of the $122 \mathrm{keV}$ peak & $<9.5 \mathrm{keV}$ \\
FWHM of the pulser peak & $<3.75 \mathrm{keV}$ \\
Leakage current drawn by each detector & $<20 \mathrm{nA}$ \\
Leakage current stability & $>3 \mathrm{hrs}$ \\
Measured $122 \mathrm{keV}$ detection efficiency & $>0.75$ \\
\hline
\end{tabular}




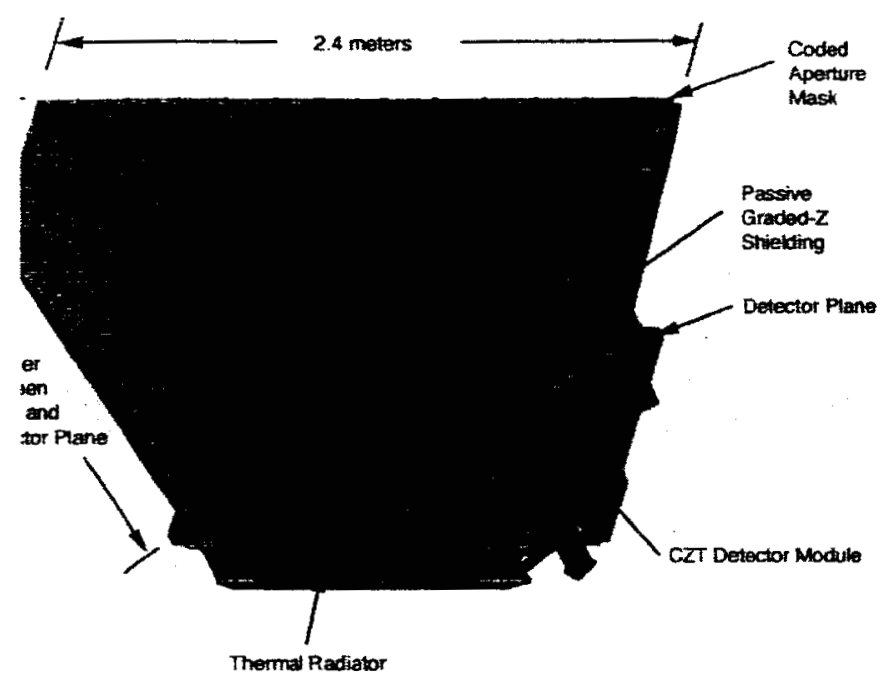

Fig. 1. The Burst Alert Telescope (BAT) on-board the Swift spacecraft. The BAT has a $3 \mathrm{~m}^{2} \mathrm{D}$-shaped coded aperture mask with $5 \mathrm{~mm}$ pixels. The $\mathrm{CdZnTe}$ array is $5,243 \mathrm{~cm}^{2}$ with $4 \mathrm{~mm}$ detectors. The Graded-Z Fringe Shield, which is not fully drawn to show the detector array, reduces the background due to cosmic diffuse emission. (NASA/GSFC)

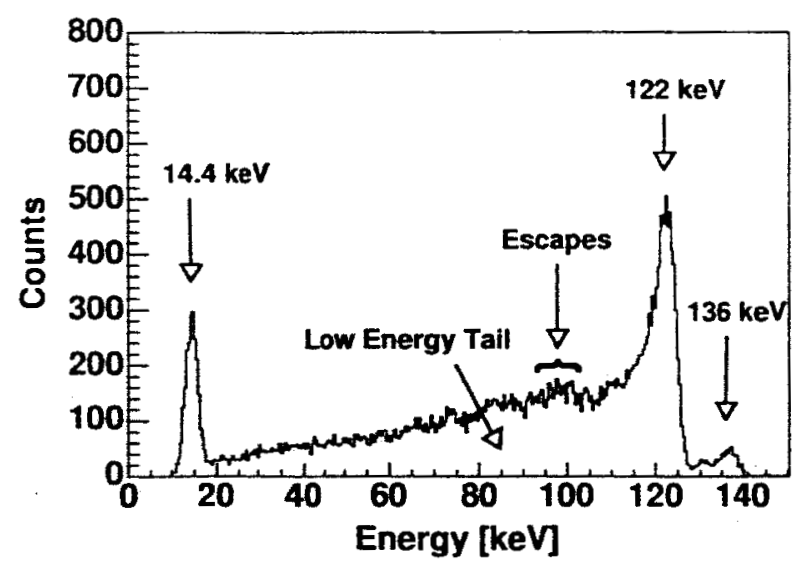

Fig. 2. A typical ${ }^{57} \mathrm{Co}$ spectrum obtained with a single $\mathrm{CdZnTe}$ detector, showing $14.4,122$ and $136 \mathrm{keV}$ lines and also a prominent low energy tail which accompanies the $122 \mathrm{keV}$ line. Small humps around $100 \mathrm{keV}$ are escape peaks associated with the $122 \mathrm{keV}$ main peak. 

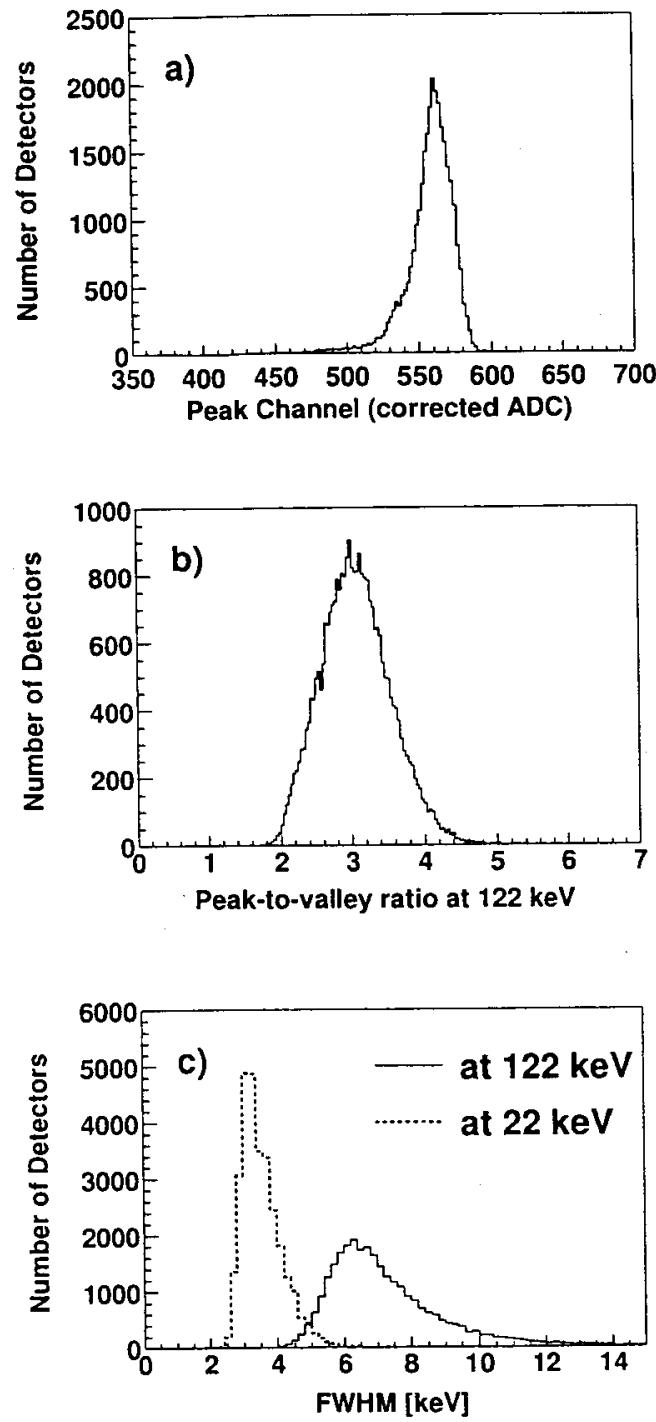

Fig. 3. Distributions of spectral parameters for $32 \mathrm{~K} \mathrm{CdZnTe} \mathrm{detectors,} \mathrm{a)} \mathrm{peak} \mathrm{channel} \mathrm{of} 122 \mathrm{keV}$ line (after corrected for electronics), b) peak-to-valley ratio at $122 \mathrm{keV}$, and c) energy resolutions (FWHM) at $122 \mathrm{keV}$ and $22 \mathrm{keV}$. 


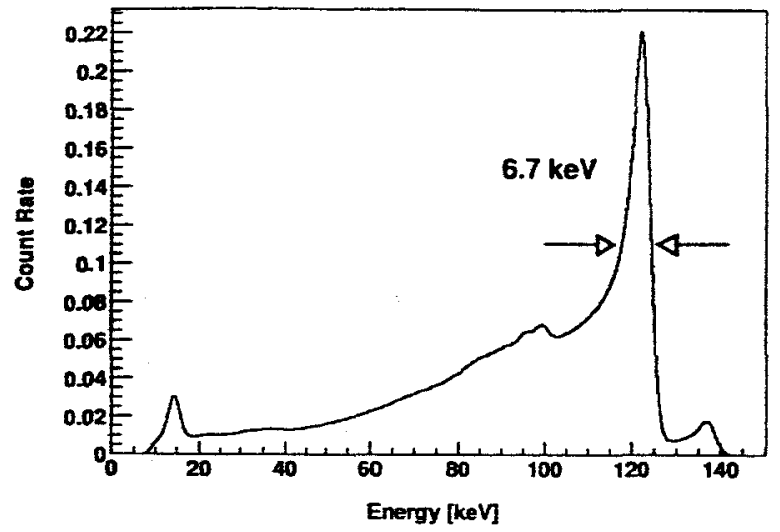

Fig. 4. Summed spectrum of $32 \mathrm{~K} \mathrm{CdZnTe} \mathrm{detectors} \mathrm{(the} \mathrm{entire} \mathrm{BAT} \mathrm{array)} \mathrm{for}{ }^{57} \mathrm{Co}$. The 122 and $136 \mathrm{keV}$ lines are clearly seen. The energy resolution is $6.7 \mathrm{keV}$ (FWHM) at $122 \mathrm{keV}$.

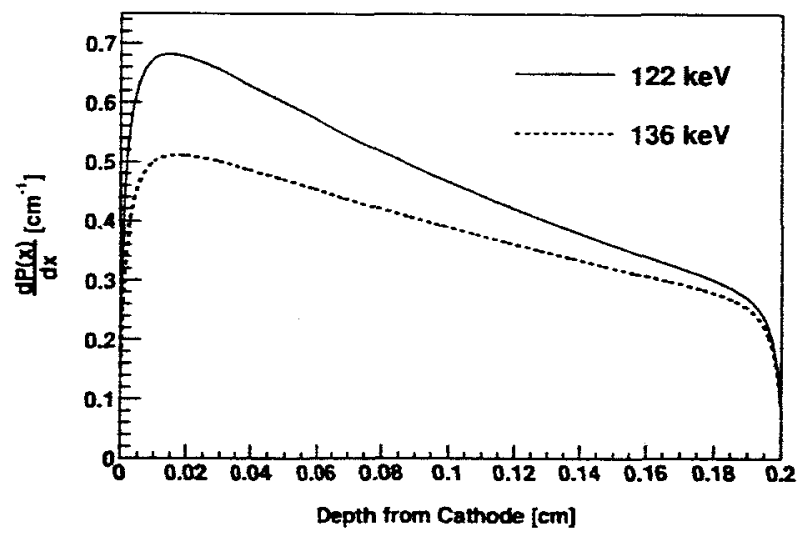

Fig. 5. The "depth distribution" for 122 and $136 \mathrm{keV}$ lines, which gives the differential probability of depositing all of the energy at various depths in a detector for an unit photon flux (photons $/ \mathrm{cm}^{2}$ ). The decreases at both ends of a detector are due to escapes from the cathode and anode surfaces. 


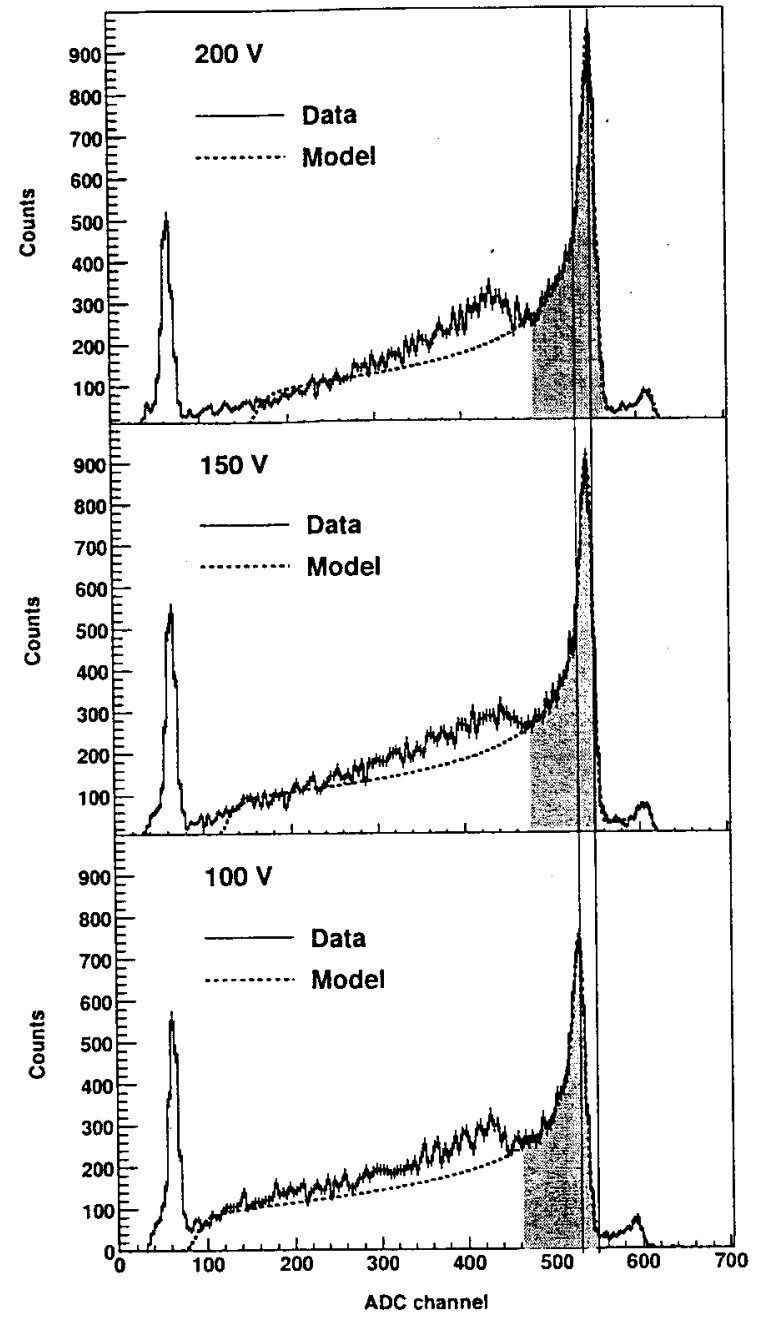

Fig. 6. The ${ }^{57} \mathrm{Co}$ spectra taken at three different bias voltages. Two guide lines are drawn to show how much the $122 \mathrm{keV}$ peak shifts with the bias voltage. The shadowed regions are fitted with the models simultaneously. The $\mu \tau$ for electrons and $\mu \tau$ for holes are determined as $2.57 \pm 0.03 \times 10^{-3}$ and $5.80 \pm 0.33 \times 10^{-5} \mathrm{~cm}^{2} \mathrm{~V}^{-1}$, respectively, with $\chi^{2} / \nu=241 / 244$. 

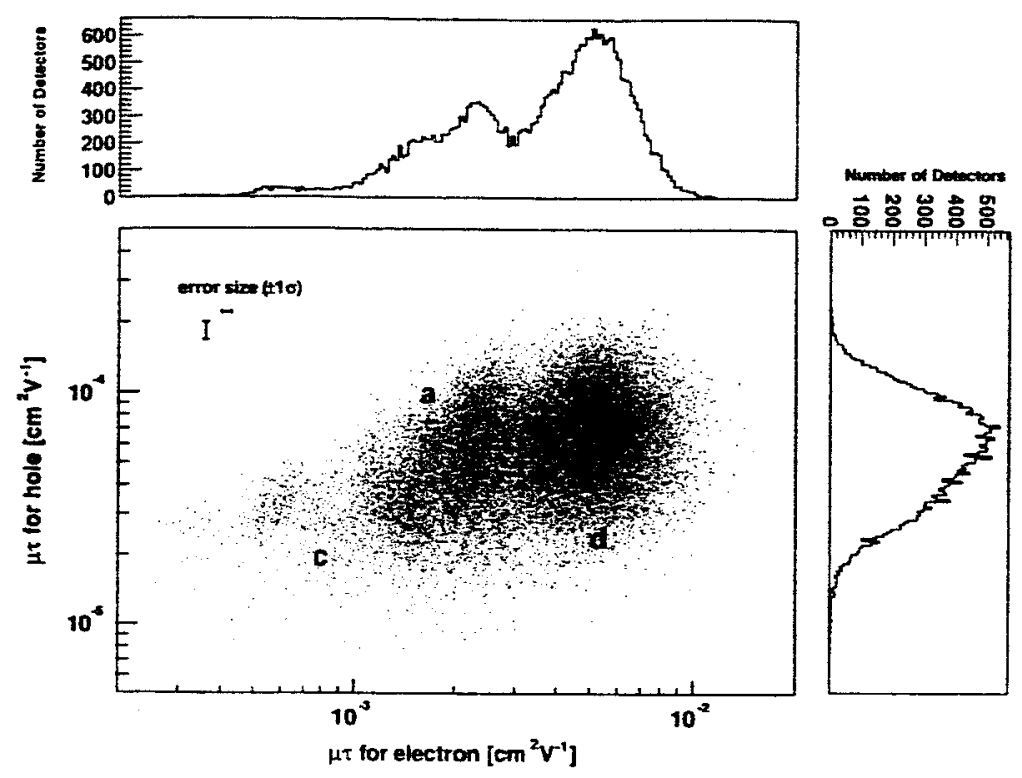

Fig. 7. A cross-plot of $\mu \tau$ for electrons and $\mu \tau$ for holes, which were determined by the spectral fitting method. The two parameters range over more than 1 order of magnitude, showing a slight correlation. The projections are also shown in the top and right panels for $\mu \tau$ for electrons and $\mu \tau$ for holes, respectively.
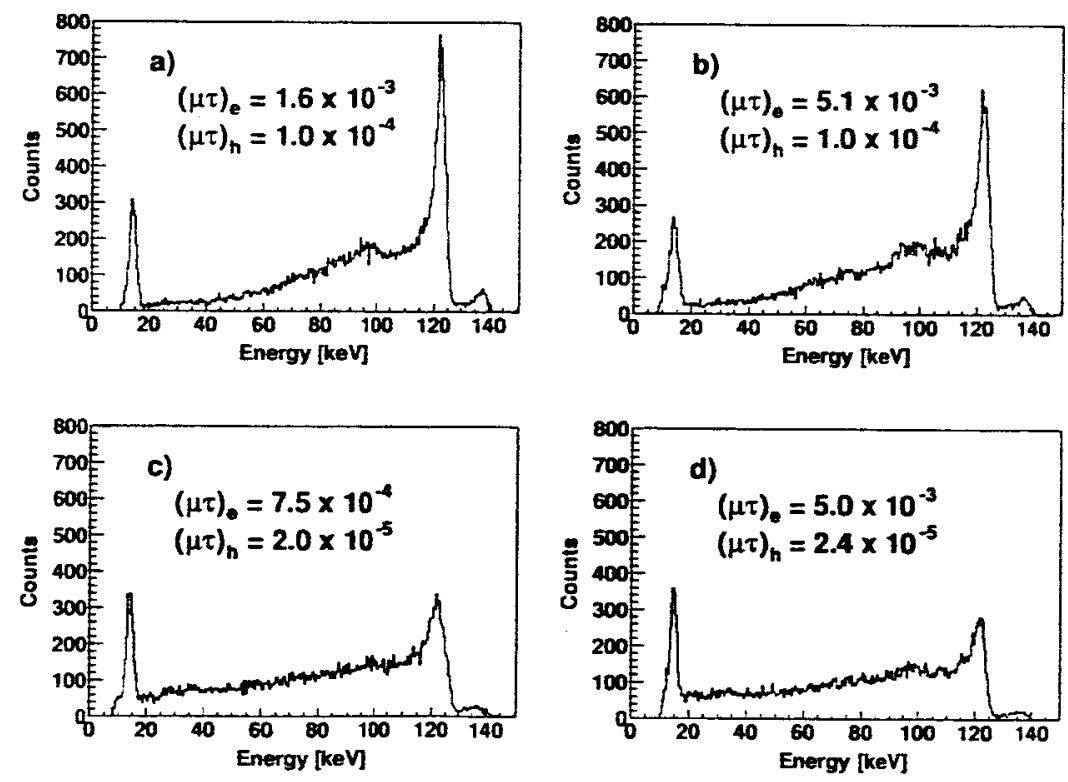

Fig. 8. Spectra obtained with single CdZnTe detectors with various sets of $\mu \tau$ products, corresponding to the symbols in the $\mu \tau$ products cross-plot (Fig.7). The detectors are exposed to a ${ }^{57}$ Co source for the same duration. 


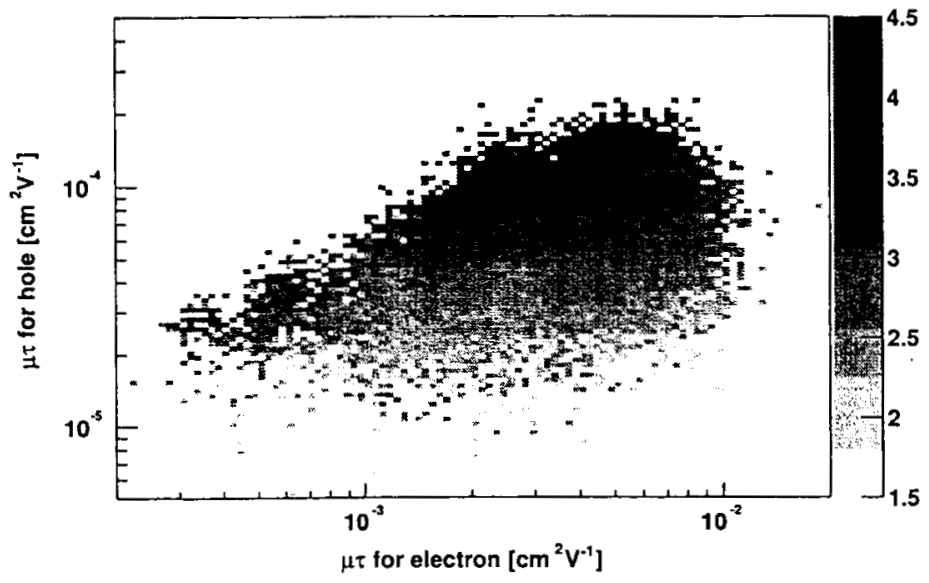

Fig. 9. The $\mu \tau$ products cross-plot divided in grids with equal intervals in log-scale, showing the measured peak-to-valley ratio averaged over the detectors in each grid in grey-scale. As the $\mu \tau$ hole becomes higher, the peak-to-valley ratio increases. The detectors with largest values of peak-to-valley ratio are found in top and left edge of the $\mu \tau$ distributions.
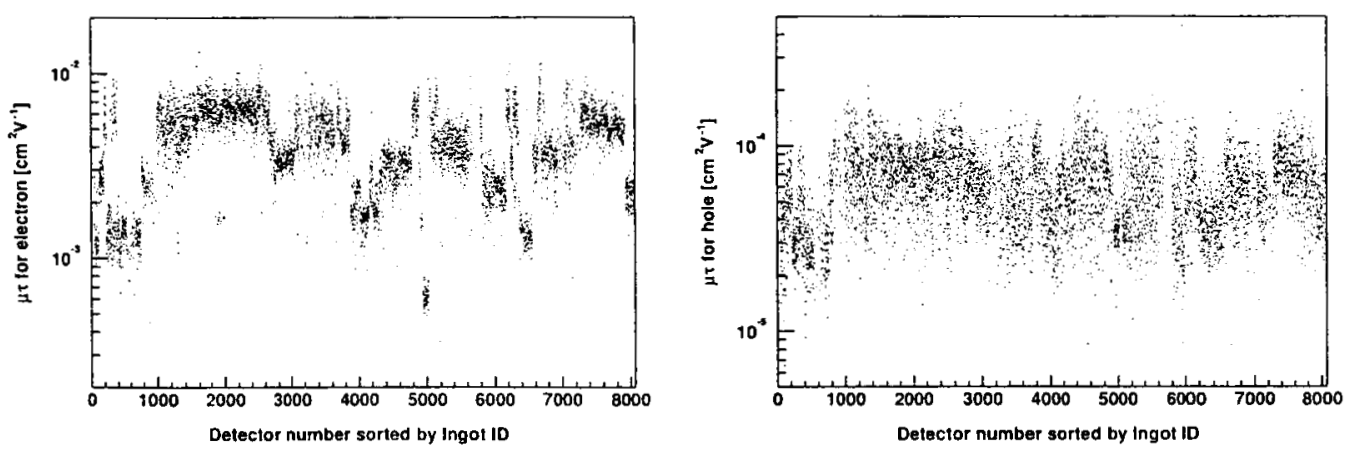

Fig. 10. The extracted $\mu \tau$ products against detector number sorted by ingot ID, indicating a strong correlation between the $\mu \tau$ products and the crystal ingots or manufacturing harness. 

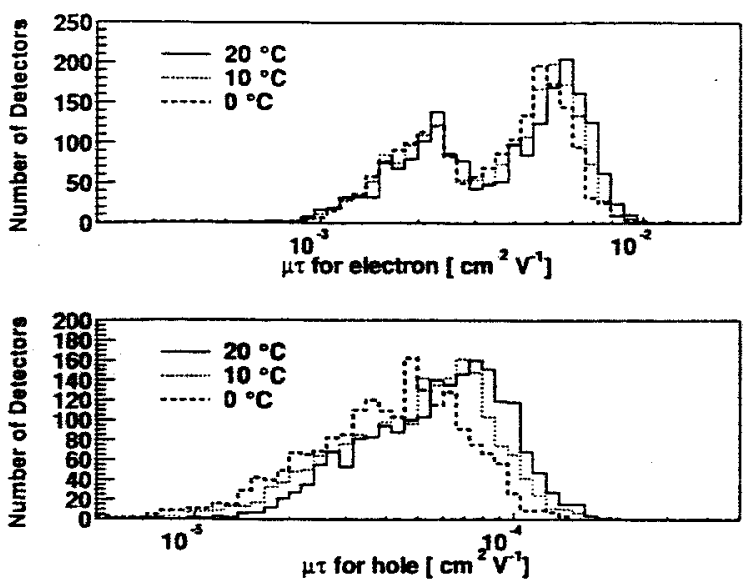

Fig. 11. The distributions of $\mu \tau$ for electrons (top) and $\mu \tau$ for holes (bottom) at three different temperatures $\left(20,10,0^{\circ} \mathrm{C}\right)$, showing trends for both distributions to shift slightly downward with decrease of temperature, but not to change the overall shape very much.
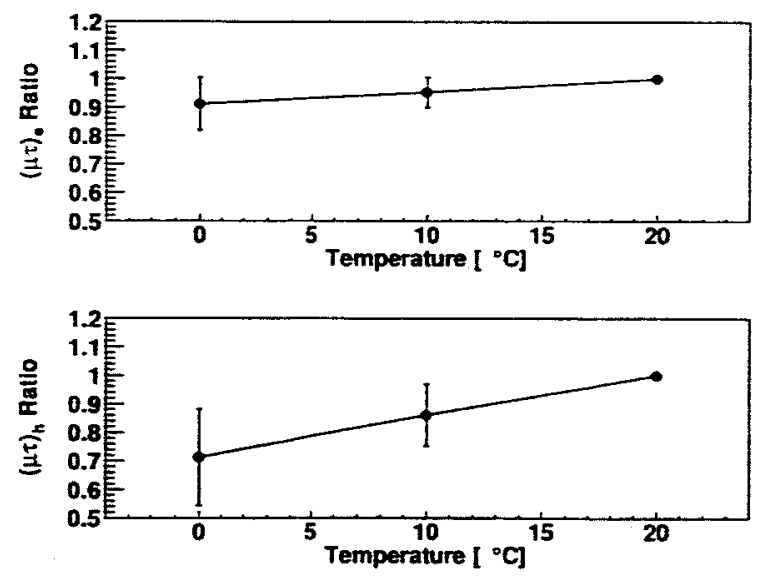

Fig. 12. For individual detectors, the relative values of $\mu \tau$ products are calculated with respect to the values at $20^{\circ} \mathrm{C}$. The average values and the RMS variations between detectors are shown for the $\mu \tau$ for electrons (top) and $\mu \tau$ for holes (bottom). 


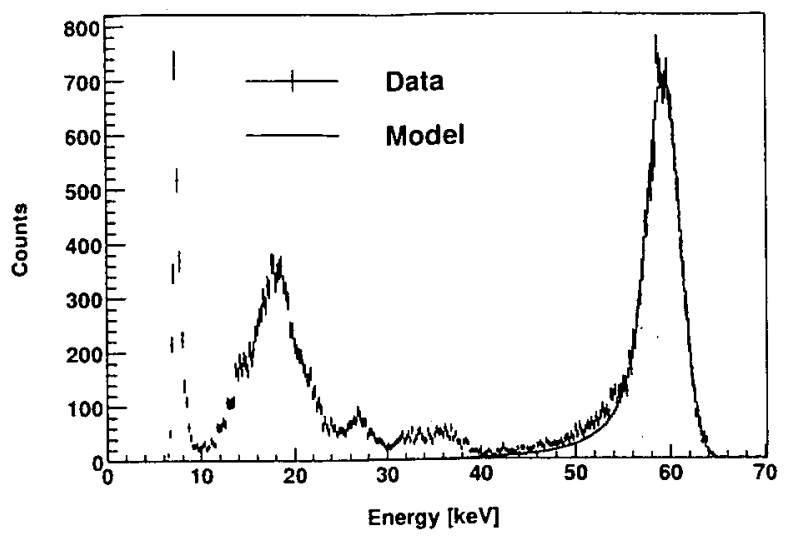

Fig. 13. An ${ }^{241} \mathrm{Am}$ spectrum obtained with a single $\mathrm{CdZnTe}$ detector, which is fitted with the model function calculated for $59.5 \mathrm{keV}$ using the values of $\mu \tau$ products derived from the spectral fitting of $122 \mathrm{keV}$ line.

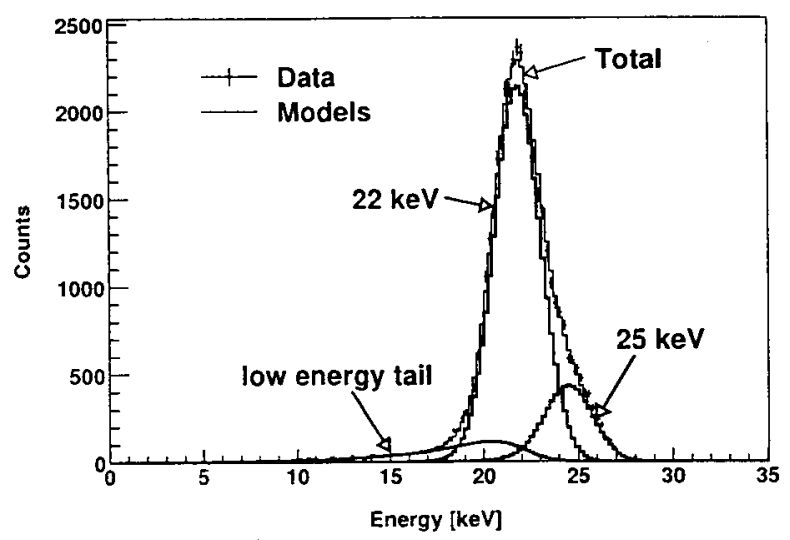

Fig. 14. A ${ }^{109} \mathrm{Cd}$ spectrum obtained with a single $\mathrm{CdZnTe}$ detector, which is fitted well with the model composed of $22,25 \mathrm{keV}$ and an additional low energy tail component.
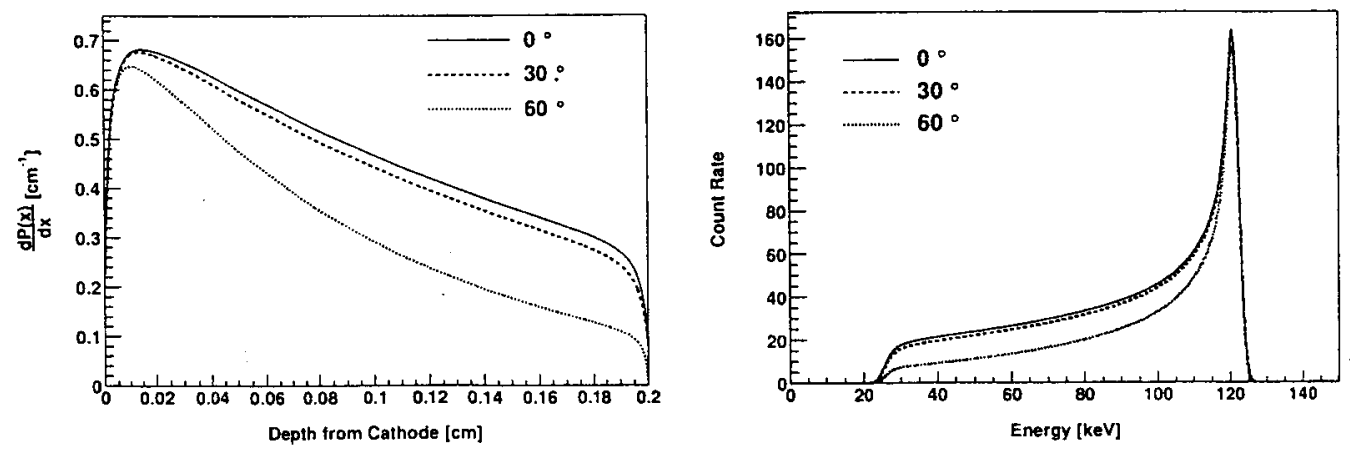

Fig. 15. The "depth distributions" for a stream of $122 \mathrm{keV}$ photons incident on a detector at three different angles $\left(0,30\right.$ and $\left.60^{\circ}\right)$ (left), the difference of which results in change of amount of tail and total area of the model spectrum (right). 

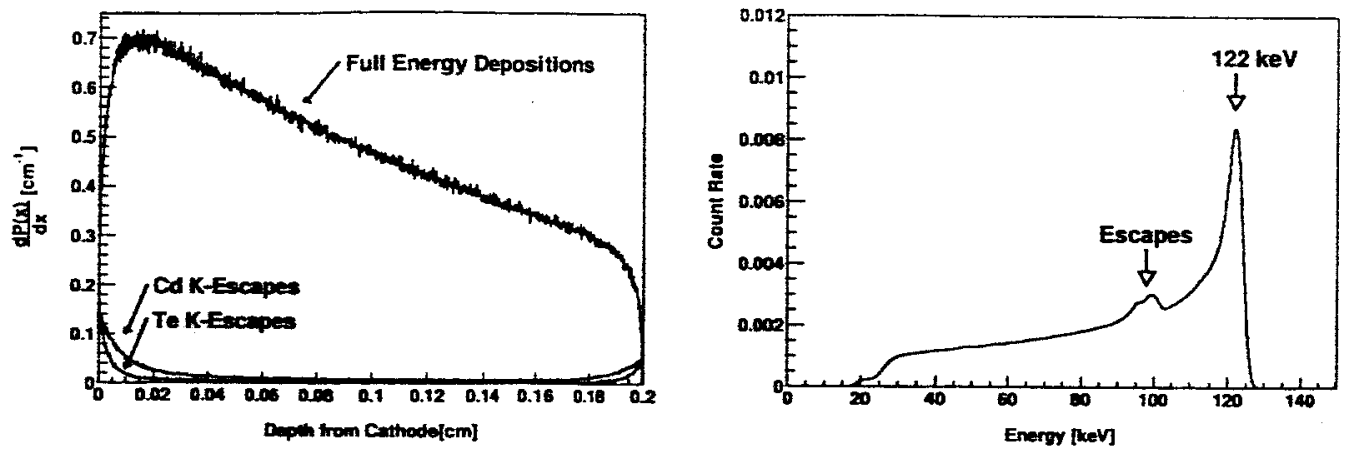

Fig. 16. The "depth distributions" for K-escapes for a stream of $122 \mathrm{keV}$ photons (left), which enables us to calculate a model spectrum including escape peaks (right).

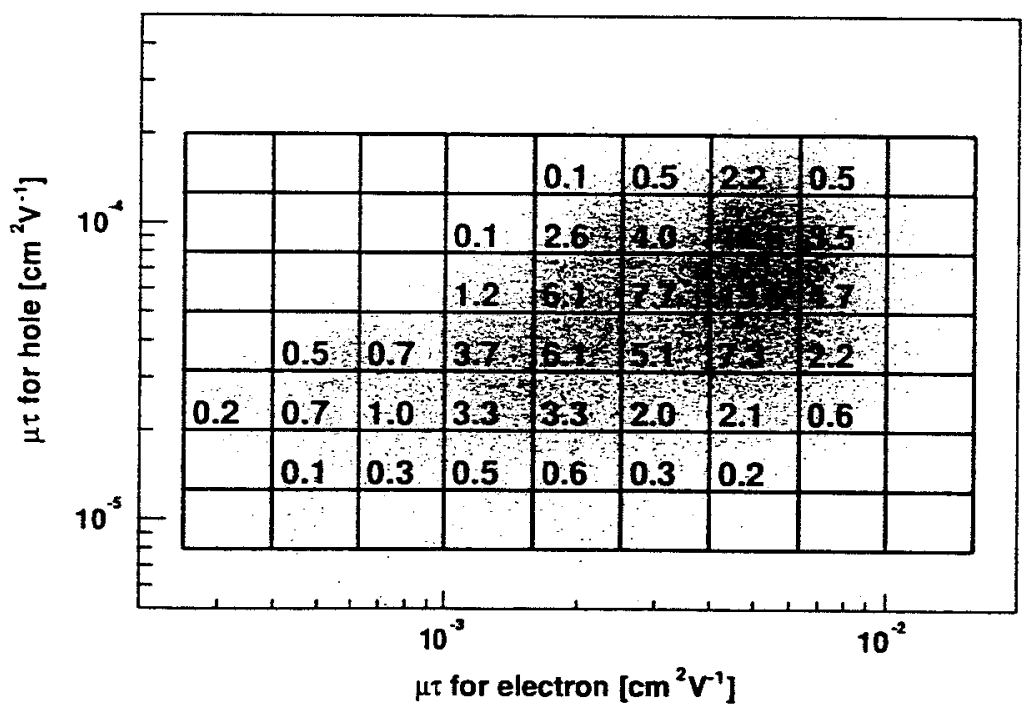

Fig. 17. The $\mu \tau$ products cross-plot divided into 9-by-7 groups with equal intervals in log-scale, showing the numbers of detectors included in each grid as percentages of the total. 


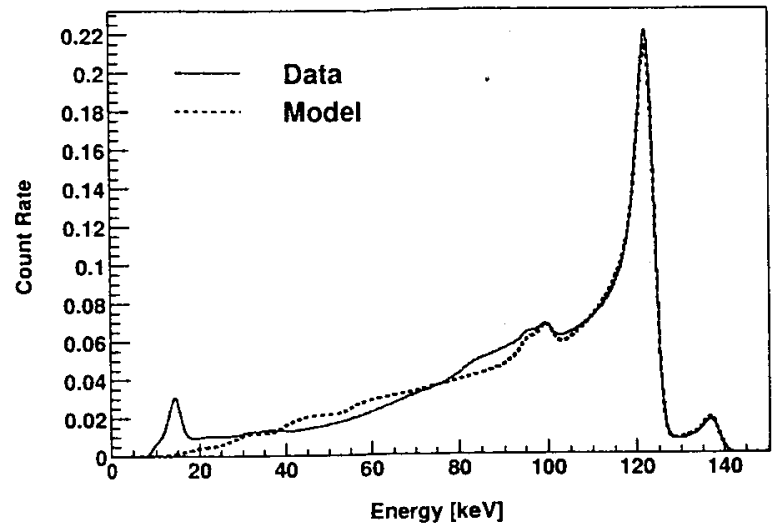

Fig. 18. A ${ }^{57}$ Co spectrum summed over the entire CdZnTe array, which is fitted with the composite spectral model for 122 and $136 \mathrm{keV}$ lines.

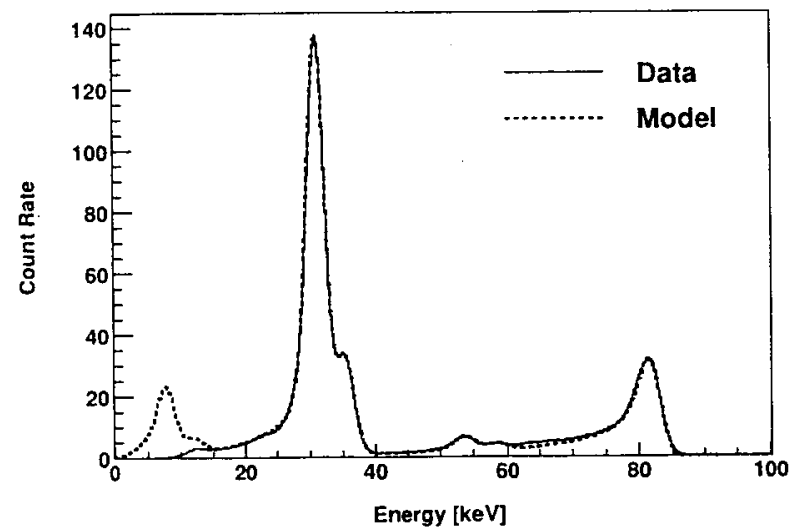

Fig. 19. $\mathrm{A}^{133} \mathrm{Ba}$ summed spectrum fitted with the composite spectral model, showing a good agreement in the wide energy range from 15 to $85 \mathrm{keV}$. lines. 